\title{
Role of defects in ultrafast charge recombination in monolayer $\mathrm{MoS}_{2}$
}

\author{
Raquel Esteban-Puyuelo $\odot$ and Biplab Sanyal ${ }^{*}$ \\ Division of Materials Theory, Department of Physics and Astronomy, Uppsala University, Box-516, Uppsala SE 75120, Sweden
}

(Received 25 March 2021; revised 11 June 2021; accepted 14 June 2021; published 28 June 2021)

\begin{abstract}
In this paper, we have systematically studied the role of point defects in the recombination time of monolayer $\mathrm{MoS}_{2}$ using time-dependent ab initio nonadiabatic molecular dynamics simulations. Various types of point defects, such as S vacancy, S interstitial, Mo vacancy, and Mo interstitial have been considered. We show that defects strongly accelerate the electron-hole recombination, especially interstitial $\mathrm{S}$ atoms do that by three orders of magnitude higher compared to pristine $\mathrm{MoS}_{2}$. Mo defects (both vacancy and interstitial) introduce a multitude of de-excitation pathways via various defect levels in the energy gap. The results of this study provide some fundamental understanding of photoinduced de-excitation dynamics in presence of defects in highly technologically relevant $2 \mathrm{D} \mathrm{MoS}_{2}$.
\end{abstract}

DOI: 10.1103/PhysRevB.103.235433

\section{INTRODUCTION}

Since the experimental realization of graphene, a single layer of graphite, in 2004 [1], two-dimensional (2D) materials are in the focus of solid-state research. Graphene has unique characteristics and has been proposed for diverse applications, in particular, as a substitute for silicon-based electronics and photovoltaics. However, the lack of a semiconducting band gap makes it unsuitable for those applications and a lot of effort has been put into finding 2D materials with similar properties and a band gap [2-4]. One path lays in considering 2D-transition metal dichalcogenides (TMDs) such as $\mathrm{MoS}_{2}$ [5-7] because they present unique electrical and optical properties and a direct band gap. From a practical point of view, 2D semiconductors have the smallest thickness possible and are thus extremely relevant in solar cells, sensing, photocatalysis, and many other applications that need miniaturization. In particular, $\mathrm{MoS}_{2}$ is technologically interesting because, unlike its bulk counterpart, it has a direct band gap of $1.8 \mathrm{eV}$ and a high electron mobility [8].

In applications such as photocatalysis, photo-induced excitation of electrons and hence creation of holes are important aspects of study especially the dynamics of chargerecombination. Nonradiative electron-hole recombination is one of the main channels for energy and carrier losses that reduce the material's efficiency. There are multiple experimental studies on $\mathrm{MoS}_{2}$, but not many theoretical advances have been made, in particular those that consider atomic scale defects $[9,10]$. Defects are very common in the fabrication of samples

\footnotetext{
*biplab.sanyal@physics.uu.se
}

Published by the American Physical Society under the terms of the Creative Commons Attribution 4.0 International license. Further distribution of this work must maintain attribution to the author(s) and the published article's title, journal citation, and DOI. Funded by Bibsam. of TMDs, with single-atom vacancies and adatoms being the most common. It is believed that defects accelerate the recombination time by introducing midgap energy levels. However, it should be noted that different types of atomic scale defects produce a variety of electronic structure with various sorts of defect levels in the energy gap. This is expected to have a profound influence on the dynamics of charge-recombination. To elucidate the role of these defects in nonradiative charge recombination, in this paper, we have studied this aspect using nonadiabatic molecular dynamics (NA-MD) simulations, currently one of the few available methods that is able to provide qualitative results for periodic systems at a reasonable computational effort.

It is extremely challenging to treat the nuclear and electronic motion fully quantum mechanically for big systems containing many electrons and nuclei. Therefore, the most popular method used is a mixed quantum-classical approach where the nuclear motion is treated classically while electrons are considered in a full quantum mechanical way. Three methods have been used for nonadiabatic molecular dynamics, viz., mean-field Ehrenfest dynamics, trajectory surface hopping, and multiple spawning [11-15]. In the trajectory surface hopping method, a number of classical trajectories are used to approximate the evolution of the nuclear wave packet. The hopping between different Born-Oppenheimer surfaces occurs from a stochastic algorithm utilizing calculated nonadiabatic coupling parameters. Details are given in the methodology section (Sec. II).

In this paper, we use $a b$ initio NA-MD within the trajectory surface hopping approach to investigate the role of neutrally charged defects (S vacancy, S interstitial, Mo vacancy, and Mo interstitial) in modifying the nonradiative recombination time in monolayer $\mathrm{MoS}_{2}$. The neutral charge state is a good representation for the $\mathrm{S}$ defects and Mo interstitial; Mo vacancy has further charged states besides the neutral $[16,17]$. We present the most probable charge-recombination pathways for each system, by pointing out which states contribute the most to the nonadiabatic coupling and thus to the transition. The paper is 
organized in the following way. We introduce in Sec. II the computational details of the electronic structure and NA-MD simulations, then we present our results for the ground-state properties in Sec. III A in absence and presence of the defects and for the nonradiative recombination in Sec. III B, followed by conclusions in Sec. IV.

\section{METHODOLOGY}

\section{A. NA-MD calculations}

This study approaches the dynamics of electrons and nuclei in a mixed quantum-classical way: the nuclear system is treated classically via adiabatic ab initio MD simulations, while the electronic degrees of freedom are treated quantum mechanically. Specifically, we used NA-MD employing basis of Slater determinants composed of single-particle timedependent (TD) adiabatic Kohn-Sham (KS) orbitals, which are computed along a given nuclear trajectory. The NA-MD trajectories were simulated using the decoherence-induced surface hopping (DISH) [18,19] within the neglect of back reaction approximation (NBRA) $[20,21]$ implemented in the PYthon eXtension for Ab Initio Dynamics (PYXAID) package [22,23]. DISH accounts for electronic decoherence by allowing hopping between potential energy surfaces at the decoherence time. This ensures a correct partitioning of the nuclear and electronic energies. The NBRA neglects the electron-nuclear back reaction effects, relying on the fact that the nuclear dynamics is driven mainly by a ground-state potential energy surface and the electronic dynamics evolves via a parametric dependence of the nonadiabatic couplings (NAC) on the nuclear positions. This reduces the number of expensive electronic-structure calculations and it has been shown to be a good approximation for large condensed-matter systems where the electron-phonon dynamics is much weaker than the electron-electron couplings and the kinetic energy is the primary source of nuclear dynamics, i.e., with a rigid structure [24-26]. Furthermore, NBRA has been previously used successfully in the study of defects in perovskites [27-30] and transition metal dichalcogenides [9,10,31], among others. It has been shown that it is a good approximation even in the presence of defects in condensed-matter systems [31], since the effect of electronic excitations to the nuclear positions is negligible when compared to thermal fluctuations.

The electronic properties of a system containing $N$ electrons can be obtained by solving the many-body timedependent Schrödinger equation (TDSE), which for the nonrelativistic case can be written as follows:

$$
i \hbar \frac{\partial \Psi}{\partial t}=\mathcal{H} \Psi
$$

where $\mathcal{H}$ is the Hamiltonian, and $\Psi\left(\vec{r}_{1}, \vec{r}_{2}, \ldots \vec{r}_{N} ; \vec{R}_{1}\right.$, $\left.\vec{R}_{2}, \ldots, t\right)$ is the all electron wave function, which depends on the positions of the electrons $\left(\vec{r}_{i}\right)$ and parametrically on the ions $\left(\vec{R}_{I}\right)$. The wave function can be expressed as a linear combination of adiabatic electronic wave functions $\psi$ and nuclear wave functions $\chi$ in the so-called Born-Oppenheimer expansion:

$$
\Psi(\vec{R}, \vec{r}, t)=\sum_{j} \psi_{j}(\vec{r} ; \vec{R}(t)) \chi_{j}(\vec{R}, t) .
$$

By inserting the Born-Oppenheimer expansion into (1), multiplying by $\psi_{i}^{*}(\vec{r} ; \vec{R})$ from the left and then integrating over the electronic coordinates $\vec{r}$ we can extract the evolution of the nuclei

$$
\begin{gathered}
{\left[T_{n u c}(\vec{R})+W_{i}(\vec{R})\right] \chi_{i}(\vec{R}, t)+} \\
\sum_{j} V_{i, j}(\vec{R}) \chi_{j}(\vec{R}, t)=i \frac{\partial}{\partial t} \chi_{i}(\{\vec{R}\}, t) .
\end{gathered}
$$

The term $W_{i}(\vec{R})=E_{i}^{e}+V_{n-n}$ is the adiabatic PES for the $i$ th electronic state and $V_{i, j}(\vec{R})$ is the hopping term that allows transitions between the $i$ th and $j$ th PES:

$$
V_{i, j}(\vec{R})=-\sum_{I} \frac{1}{2 m_{I}} G_{i, j}(\vec{R})+2 \vec{d}_{i, j}(\vec{R}) \cdot \nabla_{I},
$$

where $G_{i, j}(\vec{R})=\left\langle i\left|\nabla_{I}^{2}\right| j\right\rangle$ is the scalar coupling vector in the bracket notation and

$$
\vec{d}_{i, j}(\vec{R})=\langle i|\nabla| j\rangle
$$

is the derivative coupling matrix, more often called nonadiabatic coupling vector or NAC. Since the scalar-coupling vectors in Eq. (5) are diagonal matrix elements, they can be added to the PES to redefine the energies:

$$
\varepsilon_{i}(\vec{R})=W_{i}(\vec{R})+G_{i, j}(\vec{R}) .
$$

Solving for the nuclear degrees of freedom is complicated, and one route is to use mixed quantum-classical methods. The nuclear degrees of freedom are treated classically and the electronic problem can be solved with the stationary Schrödinger equation, for which DFT can be used:

$$
\mathcal{H}_{e l}(\vec{r}, t ; \vec{R}) \psi(\vec{r}, t ; \vec{R})=W_{i}(\vec{R}, t) \psi(\vec{r}, t ; \vec{R}) .
$$

The electronic wave function $\psi(\vec{r}, \vec{R}, t)$ is represented in the basis of stationary adiabatic functions $\phi(\vec{r} ; \vec{R}(t))$ as in the following expression

$$
\psi(\vec{r}, \vec{R}, t)=\sum_{i} c_{i}(t) \phi(\vec{r} ; \vec{R}(t)) .
$$

$c_{i}$ are the time-dependent expansion coefficients, and its evolution is governed by a TD Schrödinger equation

$$
i \hbar \frac{d c_{i}}{d t}=\sum_{j}\left(\varepsilon_{i} \delta_{i j}-i \hbar d_{i j}\right) c_{j}
$$

where $\varepsilon$ is the diagonal part of the electron Hamiltonian defined in Eq. (7) and the NACs are off-diagonal terms. In practice, the NACs are calculated between two adjacent times in Ref. [32], and more details about its implementation can be found elsewhere [22,23].

\section{B. Fewest switches surface hopping and neglect of back reaction approximation}

Equation (10) describes the coherent time evolution of electronic states coupled to the evolution of nuclear states and it can be solved with different kinds of approximations. One 
of them is to use surface hopping techniques, for example with the fewest switches surface hopping (FSSH) formulation by Tully [18], in which the time evolution of the nuclear wave functions is represented by an ensemble of trajectories that propagate via the combination of the time-dependent Schrödinger equation and stochastic factors. The electron population for all the states and and at all times in FSSH is equal to the one obtained from the TDSE, and the latter are given by the diagonal terms in the electronic density matrix

$$
\rho_{i j}=c^{*}(t) c_{j}(t) .
$$

The probability for the transition from an electronic state $|i\rangle$ to a new state $|j\rangle$ in a small enough time interval $\Delta t$ can be expressed as

$$
g_{i \rightarrow j}(t)=\max \left(0, P_{i \rightarrow j}(t)\right)
$$

with

$$
P_{i \rightarrow j}(t) \approx 2 \frac{\operatorname{Re}\left[c_{i}^{*}\left(t^{\prime}\right) c_{j}\left(t^{\prime}\right) \vec{d}_{i j}(t)\right]}{c_{i}^{*}\left(t^{\prime}\right) c_{j}\left(t^{\prime}\right)} .
$$

These probabilities are compared to a uniformly distributed random number to determine if the system is to remain in the current PES or hop to the next one and nuclear velocities are rescaled along the NAC direction to maintain the total classical electron-nuclei energy. If that rescaling is not possible, the hop is rejected.

In the original FSSH, the nuclear and electronic degrees of freedom are completely coupled, so everything is updated on the fly. However, the NBRA [22] can be used to reduce the computational cost by making the approximation that the classical trajectory of the nuclei is independent of the electronic dynamics but the electronic dynamics still depends on the nuclear positions. In practice it means that the electronic problem can be solved with a series of precomputed nuclear trajectories. Since the feedback from the electrons is not taken into account, this approximation is not valid if the electronnuclear correlations are crucial, such as in small systems like molecules. However, it is expected to produce reasonably good results for extended solids and it is currently the most widespread method to tackle solid-state systems. In FSSHNBRA, the hop rejection and velocity rescaling become

$$
g_{i \rightarrow j}(t) \longrightarrow g_{i \rightarrow j}(t) b_{i \rightarrow j}(t),
$$

where the probability is scaled by a Boltzmann factor to account for the fact that transitions to states high up in energy are less probable:

$$
b_{i \rightarrow j}(t) \begin{cases}\exp \left(-\frac{E_{j}-E_{i}-E_{\hbar \omega}}{K_{B} T}\right) & \text { if } E_{j}>E_{i}+E_{\hbar \omega} \\ 1 & \text { if } E_{j} \leqslant E_{i}+E_{\hbar \omega} .\end{cases}
$$

Here $K_{B}$ is the Boltzmann constant, $T$ is the temperature, and $E_{\hbar \omega}=\hbar \omega$ is the energy of the absorbed photon in case of light-matter interaction.

\section{Treatment of decoherence}

By having a classical description of the nuclei, the nuclear wave function has been transformed into a classical phase-space point. FSSH misses the loss of quantum coherence within the electronic subsystem that is induced by the

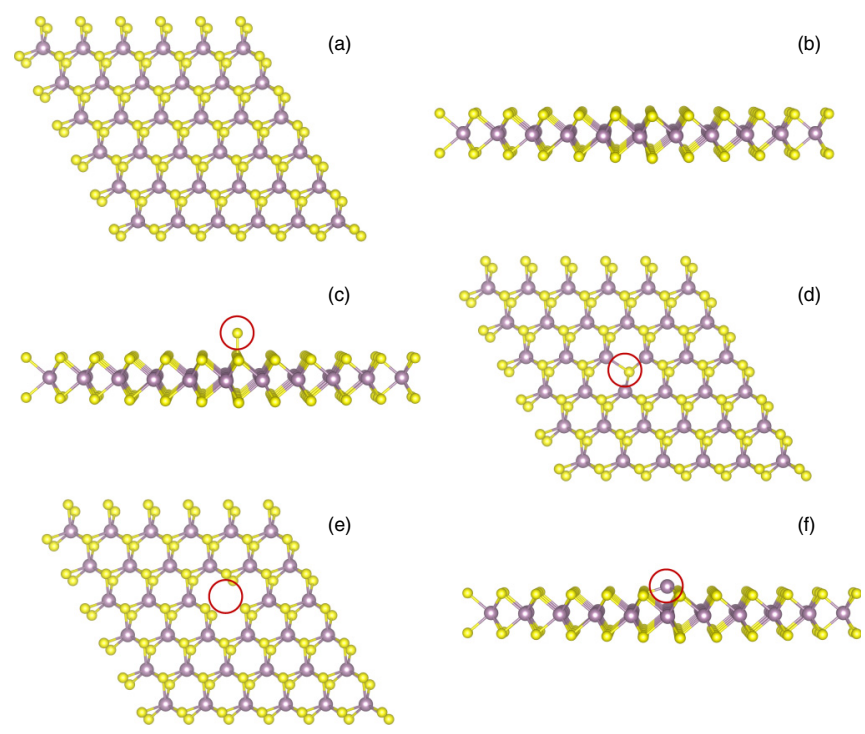

FIG. 1. Supercells of the studied $\mathrm{MoS}_{2}$ systems. S atoms are represented in yellow and Mo atoms in violet. [(a) and (b)] Top and side views of the pristine supercell respectively. [(c)-(f)] Defected structures with the defect site marked with a red circle. (c) int-S, (d) vac-S, (e) vac-Mo, and (f) int-Mo.

interaction with the quantum-mechanical vibrations and develop nonphysical coherence. Decoherence can be introduced with decoherence-induced surface hopping (DISH), which allows hops at the decoherence times only. These are calculated from the pure dephasing times, which are calculated in the optical response theory using the autocorrelation function of the energy gap fluctuation along the MD trajectory due to the nuclear motion, as described in Ref. [23].

\section{Computational details}

All the calculations have been performed with a monolayer $\mathrm{MoS}_{2}$ supercell generated by repeating the primitive cell six times in the in-plane directions. A vacuum of $20 \AA$ in the out-of-plane direction is included in order to avoid spurious interaction between periodic images of the monolayer. The $6 \times 6 \times 1$ supercell has been chosen in order to achieve the correct band folding: monolayer $\mathrm{MoS}_{2}$ has its direct band gap in the $\mathrm{K}$ point of its primitive unit cell, and it can be captured by $\Gamma$ point sampling in supercells that are commensurate with the $\mathrm{K}$ point (multiples of 3 ). The pristine supercell used in this study contains 108 atoms, and we have considered point defects of Mo and S atoms, both as vacancy and interstitial adatom, as shown in Fig. 1. The defect concentration is thus $2.75 \times 10^{13} \mathrm{~cm}^{-2}$, which is high compared to experimental conditions due to the need to find a compromise between modeling large supercells and its computational cost.

Geometry optimisations, adiabatic molecular dynamics simulations, and electronic structure calculations were performed using the plane-wave based density-functional theory based QUANTUM ESPRESSO software [33,34]. The generalized gradient approximation of Perdew, Burke, and Ernzerhof (PBE) [35] was used for the exchange-correlation functional and the core electrons were represented via scalar relativistic projector augmented Wave pseudopotentials [36] with nonlin- 

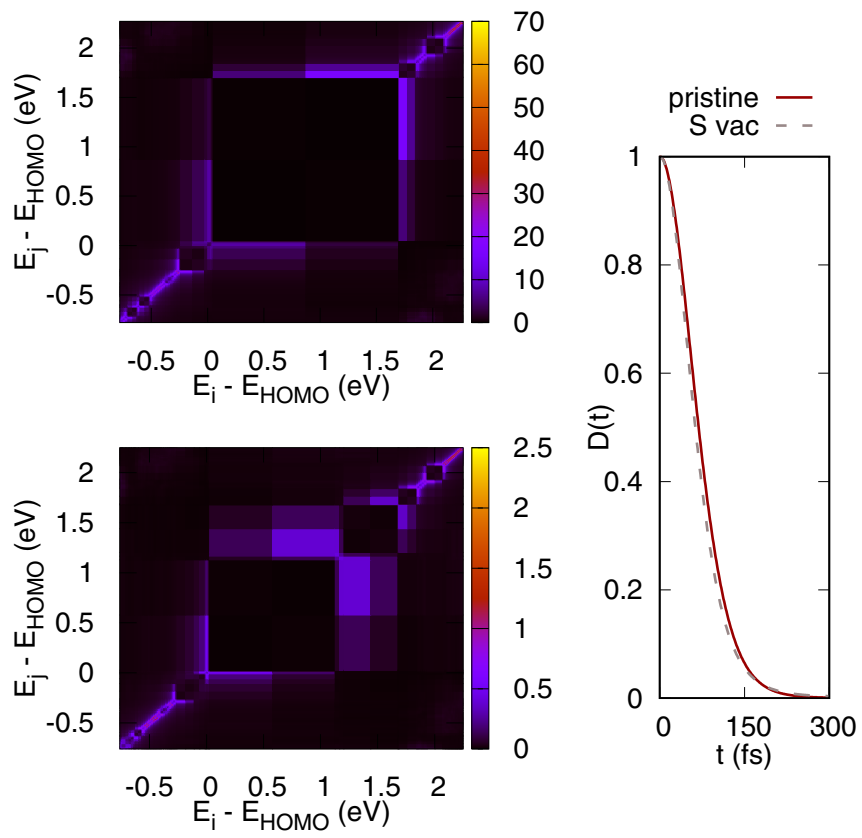

FIG. 2. Left: NAC matrices for pristine (top) and vac-S (bottom) $\mathrm{MoS}_{2}$. The 0 in the horizontal axis is the VBM and positive values stand for levels above the VBM (unoccupied) whereas negative values are for levels below the VBM (occupied). The vertical axis is defined in the same way as for the CBM. The bright color indicates the magnitude of the NAC in meV. Right: Decoherence function for the CBM-VBM transition for both systems. The decoherence time is extracted from an exponential fitting of this function.

ear core corrections. Semicore $s$ and $p$ states were included in the valence for Mo atoms. The plane wave basis size was chosen after convergence tests and was defined by a kinetic energy cutoff of $60 \mathrm{Ry}$ and a charge-density cutoff of 300 Ry. The atomic coordinates of the supercells were optimized via the BFGS quasi-Newton algorithm [37] using a fixed cell obtained from a variable cell relaxation of the $\mathrm{MoS}_{2}$ unit cell. Since it has been already shown that all the systems considered are nonmagnetic [38], all calculations were done without spin polarization.

Nuclear trajectories were produced by running a $2.5-\mathrm{ps}$ ground-state adiabatic MD simulations using the Verlet algorithm [39] with 1-fs time-step and the Andersen thermostat [40] was used to maintain ambient temperature $(300 \mathrm{~K})$. At each time step, the electronic problem was solved selfconsistently at the $\Gamma$ point. The NACs were calculated for a subset of 60-KS orbitals (30 occupied and 30 unoccupied) falling inside a 4-eV window, reasonable for optical excitations. An example of how these matrices look like is shown in Fig. 2 for pristine and vac-S $\mathrm{MoS}_{2}$.

The NA-MD trajectories were calculated for 50 initial conditions sampled from the precomputed adiabatic MD trajectory, and for each initial condition, 10000 stochastic realizations of the surface hopping process were computed. By averaging all initial conditions and stochastic realizations, the evolution of the populations of all computed states was obtained. The nonradiative relaxation time was then extracted by fitting an exponential function to the population.

\section{RESULTS AND DISCUSSIONS}

\section{A. Ground state properties}

In this section we discuss the electronic properties of pristine and defected $\mathrm{MoS}_{2}$ by analyzing the total density of states (DOS) and charge densities.

Figure 3(f) shows an energy level diagram for the systems studied in this paper: pristine, $\mathrm{S}$ vacancy (vac-S), $\mathrm{S}$ interstitial (int-S), Mo vacancy (vac-Mo), and Mo interstitial (int-Mo). The dashed lines show the position of the valence band maximum (VBM) and conduction band minimum (CBM) of the pristine system, while the short colored lines denote the actual positions of the energy levels. The occupied levels are shown in red and the unoccupied ones in green. The DOS of pristine $\mathrm{MoS}_{2}$ is shown in Fig. 3(a), where the charge density of the CBM and VBM are also shown. They match with the calculated charge density at the k-point $\mathrm{K}$ of a unit cell, as well as to previous studies [41] so the states are well captured in the supercell.

By creating defects, new states are created, mainly in the band gap, and their charge densities are plotted in Figs. 3(b)3(e) for each defected structure. Further analysis of the DOS and charge densities shows that the character of the CBM and VBM is maintained to a relatively good extent in the defected structures and therefore their charge densities are not plotted in Fig. 3, but some are shown in Fig. 4. By including an interstitial S adatom (int-S), an occupied defect level appears lying very close to the CBM but is very localized, as seen in the charge density in Fig. 3(b). It is worth noticing that the presence of this defect produces an appreciable modification in the character of the system's CBM [see Fig. 4(c)], which acquires a $p$ chalcogen character and will have an effect in the dynamics, as it will become apparent later. The next defect we considered is a $\mathrm{S}$ atom vacancy (vac-S), which creates two defect levels: an occupied defect level extremely close to the CBM and a deep unoccupied defect level. Their DOS and charge densities are included in Fig. 3(c). Mo defects create more complex energy diagrams. Since Mo atoms have more electrons than S, the energy shift of the VBM and CBM, upwards in the case of an interstitial adatom and downwards in the vacancy case, which are larger than in the case of S. Furthermore, Mo vacancy (vac-Mo) creates an occupied state close to the CBM, which induces a modification in the CBM [see Fig. 4(d)], and two unoccupied levels in the middle of the gap [Fig. 3(e)]. An interstitial Mo adatom (int-Mo) creates an occupied and two unoccupied defect levels, all three in the middle of the gap [Fig. 3(f)]. These results are in agreement with previous studies [38].

\section{B. De-excitation dynamics}

For each system, we selected the energy levels that can participate in the recombination in order to form the active space in which the DISH algorithm will be applied. For instance, in the pristine system, the VBM and CBM are doubly degenerate so the active space is formed by four orbitals and we consider excited states corresponding to the $\mathrm{CBM} \rightarrow \mathrm{VBM}$ transition, where the population of the excited state is expected to decay to the ground state. To obtain the nonradiative recombination timescale $\tau$, we fit the population increase in the ground state 

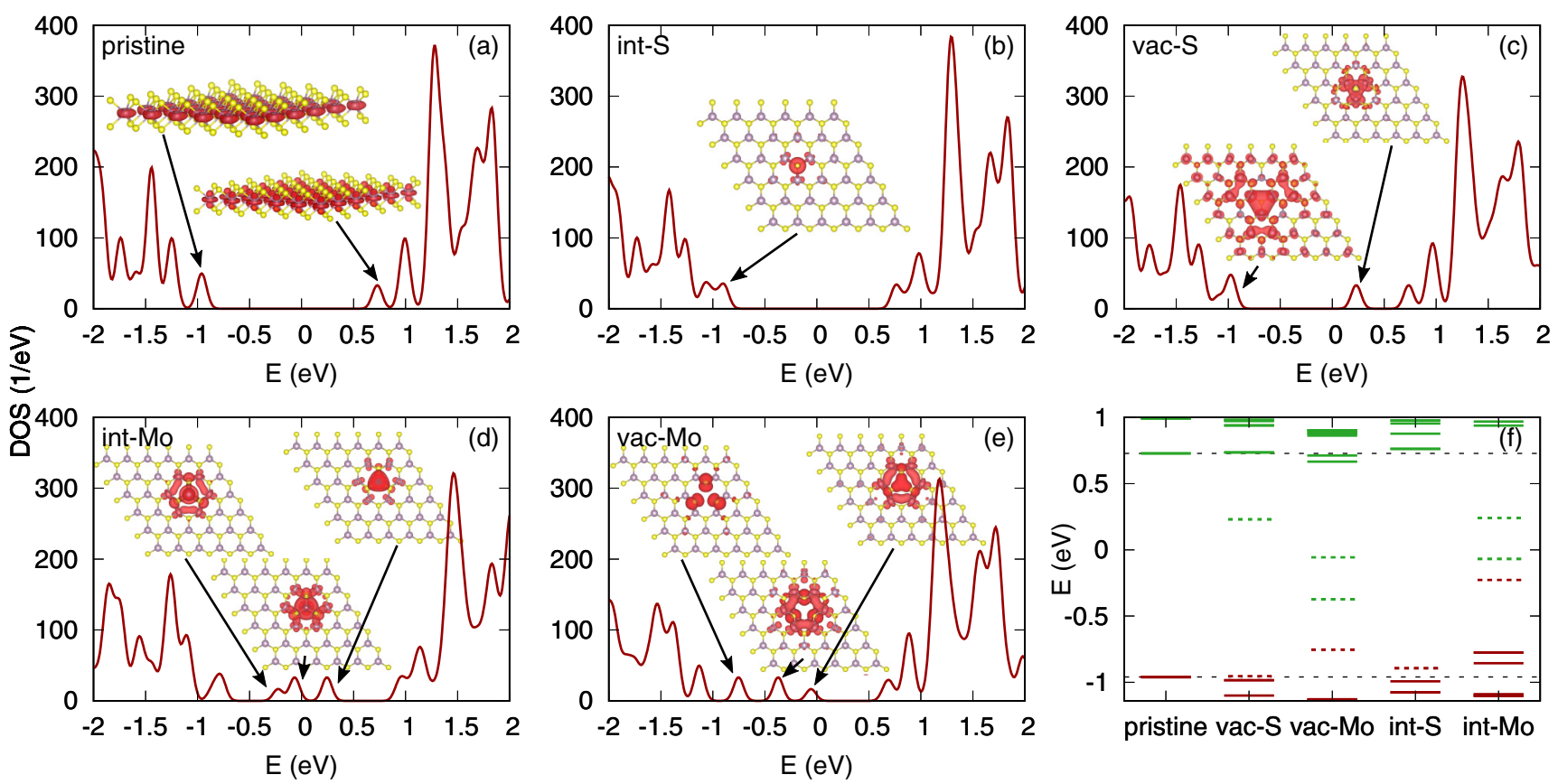

FIG. 3. [(a)-(e)] Density of states for the supercells considered. (a) Charge density of the pristine CBM and VBM as insets. [(b)-(e)] Charge density of the defect states that appear inside the pristine gap in each of the defected systems. (f) Energy level diagram for the pristine and different defected structures in $\mathrm{MoS}_{2}$. The red and green horizontal lines represent occupied and unoccupied energy levels respectively, and the dashed red and green lines indicate that those energy levels belong to occupied and unoccupied defect states, respectively, inside the gap. The dashed black lines mark where the CBM and the VBM are located in the pristine system.

with an exponential function of the form

$$
P(t)=1-\exp \left(\frac{-t}{\tau}\right),
$$

so that it reaches the normalized value of 1 at infinite time. For the defected systems, many intermediate transitions need to be considered, and a scheme of the energy levels is shown in Fig. 5. The unoccupied defects act as electron traps because excited electrons coming from the CBM can fall in these defect levels before recombining with the holes in the VBM. In the opposite way, occupied defect states act as hole traps. We consider all the transitions with all the intermediate steps. The calculated transition timescales are presented in Tables I-V. For each system, we provide the timescales for the direct nonradiative recombination and each alternative mechanism that includes transitions involving defect states. For the latter, the timescales of all the subprocesses are shown separated by commas. This is due to the fact that, although there is a clear expression for the effective time for parallel transitions

(a)

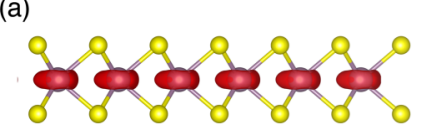

(b)
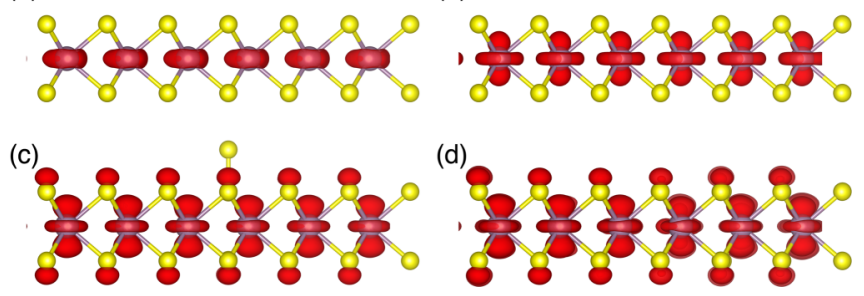

(d)

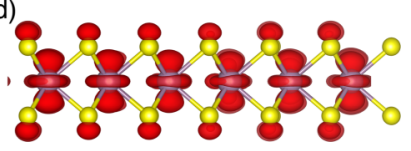

FIG. 4. In red, charge density of pristine VBM (a), pristine CBM (b). the modified CBMs of int-S (c), and vac-Mo (d). (addition of the transition rates), there is no general analytical expression for series transitions. This is the case of each of the alternative transitions to direct recombination, as for instance, (a) int-S

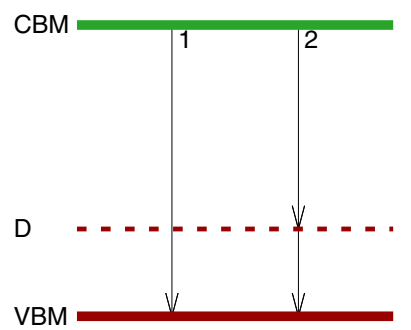

(c) int-Mo

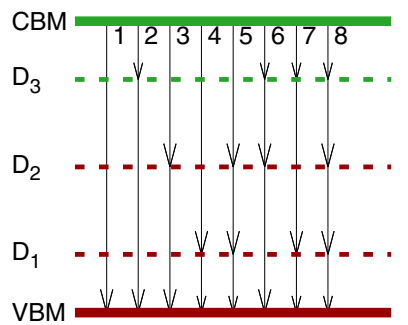

(b) vac-S

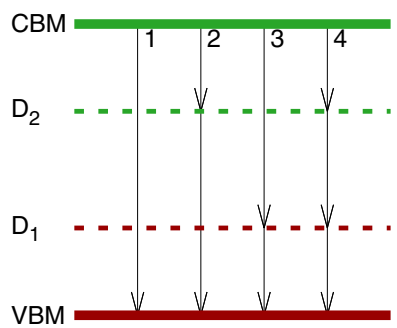

(d) vac-Mo

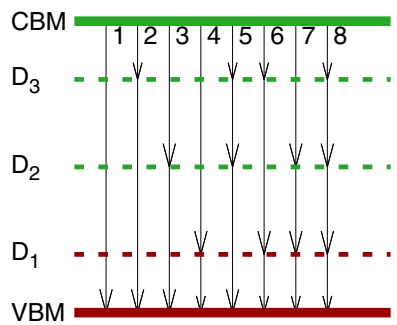

FIG. 5. Scheme of the possible transitions in (a) int-S, (b) vac-S, (c) int-Mo, and (d) vac-Mo. Red and green lines indicate occupied and unoccupied levels, respectively. Defect states inside the gap are shown with a dashed line and, within each system, they are denoted as $D_{n}$ with $n=1,2,3$ with increasing energy. 
TABLE I. Nonradiative recombination times and mechanism in pristine $\mathrm{MoS}_{2}$.

\begin{tabular}{lcc}
\hline \hline Process & Mechanism & $\tau(\mathrm{ps})$ \\
\hline 1: Direct recombination & $\mathrm{CBM} \rightarrow \mathrm{VBM}$ & 72876 \\
\hline \hline
\end{tabular}

in int-S, the process denoted by the term "hole trap" in Table II is in fact a series of transitions CBM $\rightarrow \mathrm{D} \rightarrow$ VBM [see the corresponding Fig. 5(a)].

The recombination time for pristine $\mathrm{MoS}_{2}$ is, according to our calculations, around $70 \mathrm{~ns}$, as Table I shows. Since nonradiative electron-hole recombination is the main source of energy losses in electronic devices and solar cells, this very long nonradiative recombination time is promising, since it means that efficient devices can be built from very pure $\mathrm{MoS}_{2}$ samples. However, defects clearly accelerate recombination. For instance, in systems with S adatoms (int-S, see Table II and Fig. 5a) the direct recombination time is reduced by a factor of three. This is due to the presence of the localized defect level lying close to the CBM, which distorts the CBM orbital character compared to the pristine case, as it was shown in Fig. 4. Additionally, the defect level acts as a hole trap and seems to be a much faster pathway, since the partial transitions (CBM $\rightarrow \mathrm{D}$ and $\mathrm{D} \rightarrow \mathrm{VBM})$ happen in the ps timescale. It has been shown theoretically in [38] that these defects have the lowest formation energy of all the discussed ones (around $1 \mathrm{eV}$ ), and they are also the most common defect in experimental samples grown by physical vapor deposition [42]. S vacancies (vac-S), which are present in chemical vapor deposition samples [43], are not so detrimental to the nonradiative recombination time, as shown in Table III. In fact, the direct recombination behaves in a very similar way as in the pristine sample, due to the fact that the defect levels do not distort the original CBM and VBM. It is worth noting that the transition $\mathrm{D}_{1} \rightarrow$ VBM (see Fig. 5b) is remarkably fast (1 ps), which means that the CBM and this closely lying delocalized level can be considered to overlap. On the other hand, the unoccupied defect level $\mathrm{D}_{2}$ acts as an electron trap that seems to be the fastest transition mechanism, with a timescale that is about half of the direct path.

Mo defects have higher formation energy and they are therefore less frequently observed in experiments, but it is anyhow interesting to study their relaxation dynamics. The direct nonradiative recombination in systems with interstitial Mo defects (int-Mo, see Table IV) is reduced only by a factor $1 / 3$ compared to the pristine case, which can be explained by the fact that its CBM and VBM are not appreciably modified by the presence of the multiple defect levels. Additionally, it seems that the occupied defect $\mathrm{D}_{2}$ acts as a hole trap (mech-

TABLE II. Nonradiative recombination times and mechanisms in int-S $\mathrm{MoS}_{2}$. The fastest mechanism appears in bold. For the mechanisms involving defect states, the timescales of the subprocesses are separated by commas.

\begin{tabular}{lll}
\hline \hline Process & \multicolumn{1}{c}{ Mechanism } & $\tau(\mathrm{ps})$ \\
\hline 1: Direct recombination & $\mathrm{CBM} \rightarrow \mathrm{VBM}$ & 21139 \\
2: Hole trap & $\mathrm{CBM} \rightarrow \mathrm{D} \rightarrow \mathrm{VBM}$ & 6,44 \\
\hline \hline
\end{tabular}

anism 3) that accelerates the recombination to timescales in the order of few hundreds of ps. Mechanisms 6 and 7 include multiple traps and are competing pathways and the relaxation dynamics in this system will be a combination of these three mechanisms. Mo vacancies (vac-Mo, Table V) accelerate the direct recombination by half, which is coherent with the slight modification of the CBM induced by the defects shown in Fig. 4, as it happens in the int-S system. However, the occupied defect level $\mathrm{D}_{1}$ acts as a hole trap (mechanism 1) and has a transition timescale of a few hundreds of ps, which competes with mechanism 6 (hole and electron traps 1), which includes the unoccupied $\mathrm{D}_{3}$ and has a similar timescale.

It is clear that the presence of point defects accelerates the direct nonradiative recombination in monolayer $\mathrm{MoS}_{2}$, in particular int-S does so by reducing the time to $1 / 3$ of the pristine one. However, the same defect states create alternative pathways that are always faster than their respective direct recombinations. Table VI contains a summary of the most probable mechanisms for each studied system.

In order to compare our results with experiments we can make use of the quantum yield, which is a measure of how photo-efficient a system is. In our case it can be related to the radiative $\tau_{R}^{-} 1$ and nonradiative $\tau_{N R}^{-1}$ recombination rates:

$$
Q Y=\frac{\tau_{R}^{-1}}{\tau_{R}^{-1}+\sum \tau_{N R}^{-1}} .
$$

The radiative processes involve photon emission and are typical for direct semiconductors. We distinguish between two nonradiative processes: Shockley-Read-Hall and Auger effects. In the first one, the recombination is assisted by a trap (midgap level) and can happen at low carrier concentrations both in direct and indirect semiconductors, in which lattice vibrations play an important role. Auger recombination is predominant at high carrier concentrations. The timescales that we compute with our method are nonradiative recombination times $\tau_{N R}$, and we can use (17) to compare with photoluminiscence data, which are available mostly for pristine samples. For instance, Ref. [6] reports a $Q Y$ of nearly $95 \%$ which gives a nonradiative recombination time of $11 \mathrm{~ns}$ at room temperature, in qualitative agreement with our calculations of $70 \mathrm{~ns}$ in the pristine case, and even closer in the presence of defects that reduce the effective recombination time. We have obtained longer recombination times than the ones reported in other theoretical works $[9,10]$, although we agree in the fact that $\mathrm{S}$ adatom defects (int-S) result in much faster recombination times than $\mathrm{S}$ vacancies (vac-S). Plausible reasons for our longer timescales could be their treatment of the long-term dynamics and higher density of defects due to their smaller supercell. Another explanation to the fact that we obtain longer times than the observed in experiments are excitons. It is known that excitonic effects can accelerate the recombination dynamics [44,45], and $\mathrm{MoS}_{2}$ has the well known A and B excitons. However, we would need to go beyond the one-particle picture we have used in this study to be able to capture those in a quantitative way, and it would be computationally prohibitive. Nevertheless, the one-particle picture is a fair approximation for the cases we have studied, as we have considered only the lowest excited states and they do not mix a large number of Slater determinants, 
TABLE III. Nonradiative recombination times and mechanisms in vac-S $\mathrm{MoS}_{2}$. The fastest mechanism is shown in bold. For the mechanisms involving defect states, the timescales of the subprocesses are separated by commas.

\begin{tabular}{lll}
\hline \hline Process & \multicolumn{1}{c}{ Mechanism } \\
\hline 1: Direct recombination & $\mathrm{CBM} \rightarrow \mathrm{VBM}$ & 67784 \\
2: Electron trap & $\mathrm{CBM} \rightarrow \mathrm{D}_{2} \rightarrow \mathrm{VBM}$ & 406,3440 \\
3: Hole trap & $\mathrm{CBM} \rightarrow \mathrm{D}_{1} \rightarrow \mathrm{VBM}$ & 71700,1 \\
4: Electron and hole traps & $\mathrm{CBM} \rightarrow \mathrm{D}_{2} \rightarrow \mathrm{D}_{1} \rightarrow \mathrm{VBM}$ & $406,2978,1$ \\
\hline \hline
\end{tabular}

TABLE IV. Nonradiative recombination times and mechanisms in int-Mo $\mathrm{MoS}_{2}$. The fast pathways are shown in bold. For the mechanisms involving defect states, the timescales of the subprocesses are separated by commas.

\begin{tabular}{lll}
\hline \hline Process & \multicolumn{1}{c}{ Mechanism } \\
\hline 1: Direct recombination & $\mathrm{CBM} \rightarrow \mathrm{VBM}$ & 52418 \\
2: Electron trap & $\mathrm{CBM} \rightarrow \mathrm{D}_{3} \rightarrow \mathrm{VBM}$ & 180,3251 \\
3: Hole trap 1 & $\mathrm{CBM} \rightarrow \mathrm{D}_{2} \rightarrow \mathrm{VBM}$ & 217,16 \\
4: Hole trap 2 & $\mathrm{CBM} \rightarrow \mathrm{D}_{1} \rightarrow \mathrm{VBM}$ & 4429,290 \\
5: Two successive hole traps & $\mathrm{CBM} \rightarrow \mathrm{D}_{2} \rightarrow \mathrm{D}_{1} \rightarrow \mathrm{VBM}$ & $217,2,290$ \\
6: Electron and hole traps 1 & $\mathrm{CBM} \rightarrow \mathrm{D}_{3} \rightarrow \mathrm{D}_{2} \rightarrow \mathrm{VBM}$ & $180,26,16$ \\
7: Electron and hole traps 2 & $\mathrm{CBM} \rightarrow \mathrm{D}_{3} \rightarrow \mathrm{D}_{1} \rightarrow \mathrm{VBM}$ & $180,4429,290$ \\
8: Electron and two successive hole traps & $\mathrm{CBM} \rightarrow \mathrm{D}_{3} \rightarrow \mathrm{D}_{2} \rightarrow \mathrm{D}_{1} \rightarrow \mathrm{VBM}$ & $180,26,2,290$ \\
\hline \hline
\end{tabular}

TABLE V. Nonradiative recombination times and mechanisms in vac-Mo $\mathrm{MoS}_{2}$. The fast mechanisms are shown in bold. For the mechanisms involving defect states, the timescales of the subprocesses are separated by commas.

\begin{tabular}{lll}
\hline \hline Process & \multicolumn{1}{c}{ Mechanism } & $\tau(\mathrm{ps})$ \\
\hline 1: Direct recombination & $\mathrm{CBM} \rightarrow \mathrm{VBM}$ & 32284 \\
2: Electron trap 1 & $\mathrm{CBM} \rightarrow \mathrm{D}_{3} \rightarrow \mathrm{VBM}$ & 161,742 \\
3: Electron trap 2 & $\mathrm{CBM} \rightarrow \mathrm{D}_{2} \rightarrow \mathrm{VBM}$ & 1436,74 \\
4: Hole trap & $\mathrm{CBM} \rightarrow \mathrm{D}_{1} \rightarrow \mathrm{VBM}$ & 370,116 \\
5: Two successive electron traps & $\mathrm{CBM} \rightarrow \mathrm{D}_{3} \rightarrow \mathrm{D}_{2} \rightarrow \mathrm{VBM}$ & $161,1436,116$ \\
6: Hole and electron traps 1 & $\mathrm{CBM} \rightarrow \mathrm{D}_{3} \rightarrow \mathrm{D}_{1} \rightarrow \mathrm{VBM}$ & $161,46,116$ \\
7: Hole and electron traps 2 & $\mathrm{CBM} \rightarrow \mathrm{D}_{2} \rightarrow \mathrm{D}_{1} \rightarrow \mathrm{VBM}$ & $1436,46,116$ \\
8: Hole and two successive electron traps & $\mathrm{CBM} \rightarrow \mathrm{D}_{3} \rightarrow \mathrm{D}_{2} \rightarrow \mathrm{D}_{1} \rightarrow \mathrm{VBM}$ & $161,32,370,116$ \\
\hline \hline
\end{tabular}

TABLE VI. Summary of the fastest pathways in each of the studied systems. The numbers in parentheses are related to the transitions in Fig. 5 and the respective Tables I-V.

\begin{tabular}{ll}
\hline \hline System & \\
\hline pristine & Processes \\
int-S & Hole trap (2) \\
vac-S & Electron trap (2) \\
int-Mo & Hole trap 1 (3), two successive hole traps (5), and electron and hole traps 1 (6) \\
vac-Mo & Hole trap (4), and hole and electron traps 1 (6) \\
\hline \hline
\end{tabular}


see the discussions in [44] and [46]. Therefore, we are able to describe nonradiative recombination dynamics in monolayer $\mathrm{MoS}_{2}$ with defects in a qualitative way.

We have observed that the exact transition times are sensitive to the decoherence scheme that we use, which is consistent with other similar studies (see this topics discussed in the recent review [47] and references therein). This requires special attention and will be studied in detail in near future. Furthermore, the computational method that we use in PYXAID does not enforce that the wave functions have the same phase at consecutive time steps, which would be necessary in the NAC calculation [48]. The phase inconsistency has been addressed in a recent development of Libra library [49], which will be explored in the near future. Spin-orbit coupling effects are relevant in $\mathrm{MoS}_{2}$ and have been neglected in this study due to their high computational cost, but will be addressed in future investigations.

\section{CONCLUSION}

In this paper, we have shown that point defects ( $\mathrm{S}$ and Mo adatoms and vacancies) accelerate the effective non-radiative recombination time in monolayer $\mathrm{MoS}_{2}$ samples by using NA-MD simulations. Defects have two effects in the nonradiative recombination times: first, they reduce the direct $\mathrm{CBM} \rightarrow \mathrm{VBM}$ time by as much as half compared to the pristine case; second and more importantly, they introduce midgap defect levels that allow for faster pathways for the recombination, in some case in the order of few hundreds of ps. Our calculated nonradiative recombination time of around $70 \mathrm{~ns}$ for pristine $\mathrm{MoS}_{2}$ qualitatively agrees with what has been reported experimentally. Defects, specially those involving $\mathrm{S}$ atoms, are rather common in experimental samples of monolayer $\mathrm{MoS}_{2}$. However, even if they have higher formation energy, Mo defects can be introduced controllably using helium ion beam [50]. We have shown that Mo defects introduce many more alternative pathways that accelerate recombination through the defect states, compared to $\mathrm{S}$ vacancies and adatoms, which has not been reported before. In particular, we predict that both Mo adatoms (int-Mo) and vacancies (vac-S) can accelerate the recombination in the order of hundreds of ps, and therefore are more detrimental to the material efficiency than $\mathrm{S}$ vacancies. In summary, we have analyzed the mechanisms and timescales through which nonradiative recombination can happen in realistic samples of monolayer $\mathrm{MoS}_{2}$, which is extremely important in order to move forward in the development of technology based on this material.

\section{ACKNOWLEDGMENTS}

We are thankful to Alexey V. Akimov from SUNY Buffalo (USA) for introducing us to PYXAID and helping at various steps regarding technicalities and analysis. We thank Oscar Grånäs for useful discussions. B.S. acknowledges financial support from Swedish Research Council (Grants No. 2016-05366 and No. 2017-05447) and STINT Initiation Grant Program (No. IB2015-6063). R.E.P. thanks the financial support from the Strategic Research Council (SSF) Grant No. ICA16-0037. We are grateful to NSC under Swedish National Infrastructure for Computing (SNIC), the PRACE-2IP project resource Salomon cluster based in Czech Republic at the IT4Innovations and DECI-15 project DYNAMAT for allocation of supercomputing time.
[1] K. S. Novoselov, A. K. Geim, S. V. Morozov, D. A. Jiang, Y. Zhang, S. V. Dubonos, I. V. Grigorieva, and A. A. Firsov, Science 306, 666 (2004).

[2] R. Martinazzo, S. Casolo, and G. F. Tantardini, Phys. Rev. B 81, 245420 (2010).

[3] M. Fujita, K. Wakabayashi, K. Nakada, and K. Kusakabe, J. Phys. Soc. Jpn. 65, 1920 (1996).

[4] S. Wu, S. Buckley, J. R. Schaibley, L. Feng, J. Yan, D. G. Mandrus, F. Hatami, W. Yao, J. Vučković, A. Majumdar, and X. Xu, Nature (London) 520, 69 (2015).

[5] F. Xia, H. Wang, D. Xiao, M. Dubey, and A. Ramasubramaniam, Nat. Photon. 8, 899 (2014).

[6] M. Amani, D. H. Lien, D. Kiriya, J. Xiao, A. Azcatl, J. Noh, S. R. Madhvapathy, R. Addou, K. C. Santosh, M. Dubey et al., Science 350, 1065 (2015).

[7] O. V. Yazyev and A. Kis, Mater. Today 18, 20 (2015).

[8] B. Radisavljevic, A. Radenovic, J. Brivio, V. Giacometti, and A. Kis, Nat. Nanotechnol. 6, 147 (2011).

[9] L. Li, R. Long, T. Bertolini, and O. V. Prezhdo, Nano Lett. 17, 7962 (2017).

[10] L. Li, R. Long, and O. V. Prezhdo, Nano Lett. 18, 4008 (2018).

[11] L. Wang, R. Long, and O. V. Prezhdo, Annu. Rev. Phys. Chem. 66, 549 (2015).
[12] R. Crespo-Otero and M. Barbatti, Chem. Rev. 118, 7026 (2018).

[13] T. R. Nelson, A. J. White, J. A. Bjorgaard, A. E. Sifain, Y. Zhang, B. Nebgen, S. Fernandez-Alberti, D. Mozyrsky, A. E. Roitberg, and S. Tretiak, Chem. Rev. 120, 2215 (2020).

[14] B. F. E. Curchod and T. J. Martínez, Chem. Rev. 118, 3305 (2018).

[15] L. Wang, A. Akimov, and O. V. Prezhdo, J. Phys. Chem. Lett. 7, 2100 (2016).

[16] H. P. Komsa and A. V. Krasheninnikov, Phys. Rev. B 91, 125304 (2015).

[17] A. M. Z. Tan, C. Freysoldt, and R. G. Hennig, Phys. Rev. Materials 4, 064004 (2020).

[18] J. C. Tully, J. Chem. Phys. 93, 1061 (1990).

[19] H. M. Jaeger, S. Fischer, and O. V. Prezhdo, J. Chem. Phys. 137, 22 A545 (2012).

[20] C. F. Craig, W. R. Duncan, and O. V. Prezhdo, Phys. Rev. Lett. 95, 163001 (2005).

[21] S. A. Fischer, B. F. Habenicht, A. B. Madrid, W. R. Duncan, and O. V. Prezhdo, J. Chem. Phys. 134, 024102 (2011).

[22] A. V. Akimov and O. V. Prezhdo, J. Chem. Theory Comput. 9, 4959 (2013).

[23] A. V. Akimov and O. V. Prezhdo, J. Chem. Theory Comput. 10, 789 (2014). 
[24] Z. Nie, R. Long, J. S. Teguh, C.-C. Huang, D. W. Hewak, E. K. L. Yeow, Z. Shen, O. V. Prezhdo, and Z.-H. Loh, J. Phys. Chem. C 119, 20698 (2015).

[25] A. Nijamudheen and A. V. Akimov, J. Phys. Chem. C 121, 6520 (2017).

[26] R. Long, M. Guo, L. Liu, and W. Fang, J. Phys. Chem. Lett. 7, 1830 (2016).

[27] J. He, W.-H. Fang, R. Long, and O. V. Prezhdo, J. Am. Chem. Soc. 141, 5798 (2019).

[28] W. Li, J. Liu, F. Q. Bai, H. X. Zhang, and O. V. Prezhdo, ACS Energy Lett. 2, 1270 (2017).

[29] W. Li, Y. Y. Sun, L. Li, Z. Zhou, J. Tang, and O. V. Prezhdo, J. Am. Chem. Soc. 140, 15753 (2018).

[30] W. Li, R. Long, J. Tang, and O. V. Prezhdo, J. Phys. Chem. Lett. 10, 3788 (2019).

[31] L. Li and E. A. Carter, J. Am. Chem. Soc. 141, 10451 (2019).

[32] S. Hammes-Schiffer and J. C. Tully, J. Chem. Phys. 101, 4657 (1994).

[33] P. Giannozzi, S. Baroni, N. Bonini, M. Calandra, R. Car, C. Cavazzoni, D. Ceresoli, G. L. Chiarotti, M. Cococcioni, I. Dabo et al., J. Phys.: Condens. Matter 21, 395502 (2009).

[34] P. Giannozzi, O. Andreussi, T. Brumme, O. Bunau, M. Buongiorno Nardelli, M. Calandra, R. Car, C. Cavazzoni, D. Ceresoli, M. Cococcioni et al., J. Phys.: Condens. Matter 29, 465901 (2017).

[35] J. P. Perdew, K. Burke, and M. Ernzerhof, Phys. Rev. Lett. 77, 3865 (1996).
[36] G. Kresse and D. Joubert, Phys. Rev. B 59, 1758 (1999).

[37] R. R. Fletcher, Practical Methods of Optimization, 2nd ed. (Wiley, New York, 1987).

[38] S. Haldar, H. Vovusha, M. K. Yadav, O. Eriksson, and B. Sanyal, Phys. Rev. B 92, 235408 (2015).

[39] L. Verlet, Phys. Rev. 159, 98 (1967).

[40] H. C. Andersen, J. Chem. Phys. 72, 2384 (1980).

[41] C. Autieri, A. Bouhon, and B. Sanyal, Philos. Mag. 97, 3381 (2017).

[42] J. Hong, Z. Hu, M. Probert, K. Li, D. Lv, X. Yang, L. Gu, N. Mao, Q. Feng, L. Xie, J. Zhang, D. Wu, Z. Zhang, C. Jin, W. Ji, X. Zhang, J. Yuan, and Z. Zhang, Nat. Commun. 6, 6293 (2015).

[43] W. Zhou, X. Zou, S. Najmaei, Z. Liu, Y. Shi, J. Kong, J. Lou, P. M. Ajayan, B. I. Yakobson, and J. C. Idrobo, Nano Lett. 13, 2615 (2013).

[44] B. Smith, M. Shakiba, and A. V. Akimov, J. Chem. Theory Comput. 17, 678 (2021).

[45] B. Smith, M. Shakiba, and A. V. Akimov, J. Phys. Chem. Lett. 12, 2444 (2021).

[46] A. F. Izmaylov and G. E. Scuseria, J. Chem. Phys. 129, 034101 (2008).

[47] B. Smith and A. V. Akimov, J. Phys.: Condens. Matter 32, 073001 (2020).

[48] A. V. Akimov, J. Phys. Chem. Lett. 9, 6096 (2018).

[49] A. V. Akimov, J. Comput. Chem. 37, 1626 (2016).

[50] F. Aryeetey, T. Ignatova, and S. Aravamudhan, RSC Adv. 10, 22996 (2020). 